\title{
The effect of ovarian puncture on the endocrine profile of PCOS patients who undergo IVM
}

\author{
Carolina Ortega-Hrepich ${ }^{1 *}$, Nikolaos P Polyzos ${ }^{1}$, Ellen Anckaert², Luis Guzman², Herman Tournaye', \\ Johan Smitz ${ }^{2}$ and Michel De Vos ${ }^{1}$
}

\begin{abstract}
Background: To examine whether ovarian puncture for immature oocyte retrieval and in-vitro maturation (IVM) has an effect on the endocrine profile of patients with polycystic ovary syndrome (PCOS).

Methods: Twenty-two consecutive patients with PCOS undergoing IVM treatment were included. Serum antiMüllerian hormone (AMH), sex hormone-binding globulin (SHBG), total testosterone (TT) and luteinized hormone (LH) levels were analyzed at the start of the cycle, on the day of immature oocyte retrieval (OR) and at fixed intervals thereafter, for up to three months after OR.

Results: Five days after OR circulating $\mathrm{AMH}, T$, calculated free testosterone ( $F T c)$, and LH levels were significantly reduced and circulating SHBG was significantly increased. Two weeks after OR, TT, FTC and LH remained reduced, whereas circulating AMH and SHBG levels recovered to pre-puncture values. Three months after OR, all circulating hormone levels had recovered to baseline values.
\end{abstract}

Conclusion: Ovarian puncture for the retrieval of immature oocytes and IVM in patients with PCOS has a significant impact on the ovarian endocrine profile, but this impact is brief and transient.

Keywords: Anti-Müllerian hormone, In-vitro maturation, Immature oocyte retrieval, Sex hormone-binding globulin, Total testosterone

\section{Background}

In-vitro maturation (IVM) of oocytes is a mild-approach assisted reproductive technology (ART) whereby immature oocytes are retrieved from small antral follicles. Although life birth rates after IVM are still lower than after conventional hormone-driven methods of ART that encompass oocyte maturation in vivo, IVM has promising potential in patients who suffer from significant hormonal side effects and an increased risk of ovarian hyperstimulation syndrome (OHSS) if they undergo conventional ART. Different promising strategies have been used to reduce the risk of OHSS in patients with polycystic ovary syndrome (PCOS) including ovarian stimulation using a $\mathrm{GnRH}$-antagonist protocol followed by a GnRH-agonist ovulation trigger [1], low-dose stimulation protocol using highly purified follicle-stimulation

\footnotetext{
* Correspondence: cortegah@gmail.com

'Centre for Reproductive Medicine, UZ Brussel, Laarbeeklaan 101, 1090 Brussels, Belgium

Full list of author information is available at the end of the article
}

hormone [2] and treatment segmentation with elective freezing of all oocytes or embryos [3]. Although these strategies can reduce OHSS incidence significantly, they do not completely eliminate the occurrence of OHSS in high-risk patients [4]; in these patients, IVM is a potential viable method to prevent OHSS [5]. Therefore, IVM could be highly suitable for patients with polycystic ovary syndrome.

During an IVM cycle, egg collection is typically performed using multiple transvaginal punctures of unstimulated or minimally stimulated ovaries; a recent retrospective study suggested that this egg collection procedure may exhibit a similar effect to that of laparoscopic ovarian drilling (LOD) [6]. According to this trial, oocyte retrieval for IVM was associated with an increased number of mature oocytes and embryos in subsequent in vitro fertilisation (IVF) cycles, implying that these punctures may have improved the endocrinological profile of patients with polycystic ovaries. Laparoscopic ovarian drilling (LOD) is widely used to induce ovulation 
in patients with PCOS who fail to ovulate or conceive with clomiphene citrate (CC), although there is no evidence for a significant difference in rates of clinical pregnancy, live birth, or miscarriage in women with CC resistant PCOS undergoing LOD as compared to other medical treatments [7]. Nonetheless, in spite of the hypothesis that ovarian puncture of polycystic ovaries may have a drilling-like effect, no prospective study has evaluated the effect of ovarian puncture for immature oocyte retrieval and IVM on the endocrine profile in patients with PCOS. Therefore, we performed a prospective cohort study to examine the alteration of serum antiMüllerian hormone (AMH) and other hormone levels in relation to the oocyte retrieval procedure in a consecutive series of patients with PCOS who underwent IVM treatment.

\section{Methods}

\section{Patients}

From January 2012 to July 2012, serial serum levels of endocrine parameters from 22 consecutive patients with PCOS undergoing non-hCG-triggered oocyte retrieval for IVM (IVM-OR) were prospectively analyzed. The inclusion criteria for study were as follows: women younger than 35 years old, diagnosed with PCOS undergoing IVM cycle. Patients were classified as having PCOS if they fulfilled the Rotterdam criteria for PCOS $[8,9]$. Patients with congenital adrenal hyperplasia, Cushing's syndrome and androgenic-secreting tumors were excluded. Male factor infertility was not an exclusion criterion. Written informed consent was obtained from all patients before inclusion in the trial. The study was approved by local Ethical Committee.

\section{IVM cycle characteristics}

In oligomenorrhoeic and amenorrhoeic patients, a withdrawal bleeding was induced using oral dydrogesterone (Duphaston ${ }^{\circ}$, Abott Products GmbH, Hannover, Germany; $10 \mathrm{mg}$ daily for 5 days). All patients received transdermal 17ß-estradiol gel (Oestrogel ${ }^{\circ}$, Besins Healthcare, Paris, France) for endometrial preparation, at a starting dose of $4 \mathrm{mg}$ daily for seven consecutive days, after which the dose was increased to $7.5 \mathrm{mg}$ daily. After a mean interval of $10.7 \pm 2.6$ days of $17 \beta$-estradiol gel administration all patients received 150 IU highly purified human menopausal gonadotropin (HP-hMG) (Menopur'; Ferring Pharmaceuticals A/S, Copenhagen, Denmark) daily for three consecutive days. Transvaginal oocyte retrieval was scheduled 42 hours after the last injection of HPhMG and was performed using a single lumen 17 GA needle (Cook Medical, K-OPS-1230-VUB, Limerick, Ireland) at an aspiration pressure of $70 \mathrm{~mm} \mathrm{Hg}$. To avoid the retrieval of oocytes at divergent stages of maturation at the time of retrieval, no human chorionic gonadotropin
(hCG) trigger was administered. Cumulus-oocyte complexes (COCs) were matured for 40 hours in MediCult IVM $^{\circ}$ System (Origio, Måløv, Denmark). Intracytoplasmic sperm injection (ICSI) and embryo transfer were performed as previously described $[10,11]$.

\section{Measurements and follow-up}

Serial blood sampling for the analysis of serum levels of $\mathrm{AMH}$, total testosterone (TT), calculated free testosterone (FTc), sex hormone-binding globulin (SHBG), follicle stimulating hormone (FSH) and luteinized hormone (LH) was performed on day two of the IVM treatment cycle, on the day of IVM-OR (before the oocyte collection), five days after IVM-OR and two weeks after IVMOR.

To extend the interval of follow-up after the IVM cycle, patients were invited to return to the clinic for further blood sampling three months after oocyte retrieval and a subanalysis was performed in those who did return.

\section{Assays}

Serum AMH levels were measured with the Beckman Coulter Gen II ELISA kit (Beckman Coulter, Marseille, France). To circumvent inter-assay variability, all samples were processed simultaneously. Serum FSH, LH, estradiol (E2), progesterone, SHBG and total testosterone levels were measured on a Cobas 6000 immunoanalyser (Roche Diagnostics, Mannheim, Germany). Free testosterone was calculated from total serum testosterone and SHBG as measured by immunoassay and assuming a fixed albumin concentration as described previously [12].

\section{Statistical analysis}

Data were analyzed using SPSS 20.0 statistical software. Normality of the distribution for each of the outcomes was examined using the Shapiro-Wilk test. Due to the lack of normality in the distribution, paired analysis was performed using the Wilcoxon Signed-Rank Test. Differences were considered statistically significant when $\mathrm{P} \leq 0.05$.

\section{Results}

Table 1 shows baseline patient and hormonal characteristics of the study population.

\section{Circulating hormone levels during the IVM cycle}

On day two of the cycle $(\mathrm{cd} 2)$, the mean basal levels of serum AMH, E2, TT, FTc and SHBG were $8.9 \pm 4.0 \mu \mathrm{g} / \mathrm{L}$, $47.4 \pm 13.1 \mathrm{ng} / \mathrm{L}, 0.36 \pm 0.13 \mathrm{nmol} / \mathrm{L}, 5.0 \pm 3.6 \mathrm{pmol} / \mathrm{L}$ and $74.6 \pm 59.1 \mathrm{nmol} / \mathrm{L}$, respectively. After endometrial priming with transdermal $17 ß$-estradiol and a cumulative dose of $450 \mathrm{IU}$ HP-hMG, a second blood sample was obtained on the day of IVM-OR: serum AMH levels were significantly reduced to $7.5 \pm 3.2 \mu \mathrm{g} / \mathrm{L}(\mathrm{p}=0.005)$ and serum E2 
Table 1 Baseline patient characteristics

\begin{tabular}{ll}
\hline & Mean \pm SD \\
\hline Age $(\mathrm{y})$ & $29.7 \pm 3.5$ \\
$B M I\left(\mathrm{~kg} / \mathrm{m}^{2}\right)$ & $30.3 \pm 9.0$ \\
AFC & $20.2 \pm 11.0$ \\
FSH (IU/L) & $5.5 \pm 1.2$ \\
E2 $(\mathrm{ng} / \mathrm{L})$ & $47.3 \pm 13.1$ \\
LH (IU/L) & $7.9 \pm 4.4$ \\
Progesterone $(\mu \mathrm{g} / \mathrm{L})$ & $0.6 \pm 0.2$ \\
\hline
\end{tabular}

$B M I$ body mass index, $A F C$ antral follicle count, $F S H$ follicle stimulating hormone, E2 Estradiol, LH Luteinizing hormone.

and TT levels were significantly increased to $1098 \pm$ $1831 \mathrm{ng} / \mathrm{L}(\mathrm{p}<0.001), 0.48 \pm 0.21 \mathrm{nmol} / \mathrm{L}(\mathrm{p}=0.002$, resp). FTc, SHBG and LH levels all remained unchanged on the day of OR compared to basal cd2 values $[5.3 \pm 2.9 \mathrm{pmol} / \mathrm{L}$ $(\mathrm{p}=0.570), \quad 85.1 \pm 48.3 \mathrm{nmol} / \mathrm{L}(\mathrm{p}=0.082)$ and $10.2 \pm$ $7.8 \mathrm{IU} / \mathrm{L}, \mathrm{p}=0.277$, resp.].

Five days after IVM-OR, circulating AMH levels significantly dropped to $6.3 \pm 3.2 \mu \mathrm{g} / \mathrm{L}(\mathrm{p}=0.001)$ compared to values at IVM-OR and circulating levels of TT, FTc and LH levels were also significantly reduced $[0.28 \pm$ $0.12 \mathrm{nmol} / \mathrm{L}(\mathrm{p}<0.001), 2.9 \pm 1.8 \mathrm{pmol} / \mathrm{L}(\mathrm{p}<0.001)$ and $5.8 \pm 4.1 \mathrm{IU} / \mathrm{L}(\mathrm{p}=0.023)$, resp.]. Circulating SHBG was significantly increased to $99.4 \pm 57.3 \mathrm{nmol} / \mathrm{L}(\mathrm{p}=0.017)$.

These significant changes of circulating AMH and SHBG levels were no longer observed two weeks after OR: circulating $\mathrm{AMH}$ recovered to $7.6 \pm 3.6 \mu \mathrm{g} / \mathrm{L}(\mathrm{p}=0.910)$ and SHBG recovered to $81.6 \pm 54.4 \mathrm{nmol} / \mathrm{L}(\mathrm{p}=0.875)$. However, TT, FTc and LH remained significantly reduced two weeks after IVM-OR, compared to levels at IVM-OR $[0.22 \pm 0.1 \mathrm{nmol} / \mathrm{L}(\mathrm{p}=0.001), 2.7 \pm 1.5 \mathrm{pmol} / \mathrm{L}(\mathrm{p}=0.002)$ and $3.7 \pm 3.0 \mathrm{IU} / \mathrm{L}$ ( $\mathrm{p}=0.008$ ), resp.] (Table 2).

Because of the significant impact of IVM-OR on serum androgens and LH levels two weeks after IVMOR, patients were invited to have further blood sampling for hormone analysis three months after IVM-OR.

\section{Circulating hormone levels three months after IVM-OR}

Blood samples for hormone analysis were obtained from five patients at three months' follow-up. The remaining
17 patients were unavailable for this subanalysis: 10 patients had undergone a second IVM-OR procedure in the meantime, 2 patients were receiving hormonal treatment, 3 patients had become pregnant after the IVM treatment cycle and 2 patients were lost to follow-up.

Three months after IVM-OR, no significant differences were found when comparing circulating levels of $\mathrm{AMH}$, TT, FTc, SHBG and LH to basal levels ${ }^{(a)}$ or OR levels ${ }^{(b)}$. Total testosterone, FTc and LH levels had recovered to $0.37 \pm 0.16 \mathrm{nmol} / \mathrm{L}\left(\mathrm{p}=0.225^{(\mathrm{a})}, \mathrm{p}=0.5^{(\mathrm{b})}\right), 4.0 \pm 2.4 \mathrm{pmol} / \mathrm{L}$ $\left(\mathrm{p}=0.893^{(\mathrm{a})}, \mathrm{p}=0.893^{(\mathrm{b})}\right)$ and $5.8 \pm 4.7 \mathrm{IU} / \mathrm{L}\left(\mathrm{p}=0.345^{(\mathrm{a})}\right.$, $\mathrm{p}=0.686^{(\mathrm{b})}$ ), respectively (Table 2 ).

\section{Discussion}

To our knowledge, this is the first prospective study to examine the effect of ovarian puncture for retrieval of immature oocytes on the endocrine profile in patients with PCOS undergoing IVM treatment. The results of our study suggest that the effect of oocyte retrieval of immature oocytes on the endocrine profile of these patients is significant but transient and that ovarian puncture for IVM does not offer long-term prospects of an alteration of the endocrine profile in patients with PCOS, as observed after LOD. Although the exact mechanism of action of LOD is unknown, it is likely that multiple ovarian punctures may destroy ovarian androgen-producing tissue and by doing so result in reduced peripheral conversion of androgen into oestrogen [13]. This is further documented by the observation that LOD can restore ovulation, results in good pregnancy rates $[14,15]$ and significantly reduces AMH levels [16], LH/FSH ratio, serum concentration of $\mathrm{LH}$, testosterone and free androgen index. The effect of LOD on these endocrine parameters often persists for many years after the procedure [17].

Although gonadotropin doses in IVM cycles are typically much lower than those administered in conventional ART (c-ART) cycles, clinical protocols for IVM often include mild stimulation using low-dose gonadotropins and/or oral or transdermal estrogens [18-20] to increase endometrial thickness. In our study, patients received $14.7 \pm 2.5$ days of transdermal oestradiol and 450 IU HPhMG cumulatively for endometrial priming before

Table 2 Endocrine profile after oocyte retrieval (OR) for IVM in PCOS

\begin{tabular}{lllll}
\hline & Basal & $\begin{array}{l}\text { OR day } \\
\text { (All values compared to basal values) }\end{array}$ & $\begin{array}{l}\mathbf{5} \text { days after OR } \\
\text { (All values compared to OR values) }\end{array}$ & $\begin{array}{l}\mathbf{2} \text { weeks after OR } \\
\text { (All values compared to OR values) }\end{array}$ \\
\hline AMH $(\mu \mathrm{g} / \mathrm{L})$ & $8.9 \pm 4.0$ & $7.5 \pm 3.2^{*}$ & $6.3 \pm 3.2^{*}$ & $7.6 \pm 3.6^{\mathrm{NS}}$ \\
$T \mathrm{~T}(\mathrm{nmol} / \mathrm{L})$ & $0.36 \pm 0.13$ & $0.48 \pm 0.1^{*}$ & $0.28 \pm 0.12^{* *}$ & $0.22 \pm 0.1^{*}$ \\
$\mathrm{FTC}(\mathrm{pmol} / \mathrm{L})$ & $5.0 \pm 3.6$ & $5.3 \pm 2.9^{\mathrm{NS}}$ & $2.9 \pm 1.8^{* *}$ & $2.7 \pm 1.5^{*}$ \\
$\mathrm{SHBG}(\mathrm{nmol} / \mathrm{L})$ & $74.6 \pm 59.1$ & $85.1 \pm 48.3^{\mathrm{NS}}$ & $99.4 \pm 57.3^{*}$ & $81.6 \pm 54.4^{\mathrm{NS}}$ \\
$\mathrm{LH}(\mathrm{IU} / \mathrm{L})$ & $7.9 \pm 4.4$ & $10.2 \pm 7.8^{\mathrm{NS}}$ & $5.8 \pm 4.1^{*}$ & $3.7 \pm 3.0^{*}$ \\
\hline
\end{tabular}

Values represent mean and SD.

$A M H$ Antimüllerian hormone, $\pi$ total testosterone, SHBG sex hormone-binding globulin, $F T C$ calculated free testosterone, $L H$ Luteinizing hormone, FSH Follicle-stimulating hormone. ${ }^{*} \mathrm{P}<0.05 ;{ }^{* *} \mathrm{P}<0.001$; NS not statistically different. 
oocyte retrieval, resulting in mean serum E2 levels $(1,098 \pm 1,831 \mathrm{ng} / \mathrm{L})$ on the day of OR-IVM. The observation that serum AMH levels were significantly reduced on the day of IVM-OR ( $\mathrm{p}=0.005)$ is in accordance with previous studies showing reduced AMH levels after controlled ovarian stimulation for c-ART [21,22]. Nevertheless, although in c-ART cycles the number of small antral follicles producing $\mathrm{AMH}$ decreases as follicles progressively grow in response to FSH stimulation, this mechanism does not occur in IVM-cycles: in the study presented here, IVM-OR was performed when follicles had a maximal mean diameter of $10 \mathrm{~mm}$. Because previous studies have shown that FSH increases estradiol levels and suppresses AMH secretion [23,24], we propose that the observed significant reduction of circulating $\mathrm{AMH}$ levels on the day of IVM-OR is caused by increased serum estradiol levels secondary to exogenous administration of estradiol and gonadotropins. Elevated estradiol levels also enhance hepatic SHBG synthesis and secretion, resulting in increased SHBG levels on the day of IVM-OR. TT values were also increased after mild ovarian stimulation for IVM $(\mathrm{p}=0.002)$. This might be explained by the fact that the amounts of $\mathrm{LH}$ and hCG present in HP-hMG formulations are capable of stimulating androgen substrate production from theca cells [25].

Taking into account that decreased AMH biosynthesis following LOD restores ovulatory function through enhanced follicular sensitivity to circulating FSH levels, we speculated that a similar effect might occur following immature oocyte retrieval from small follicles. LOD has been proven to significantly reduce circulating $\mathrm{AMH}$ levels [26], with values remaining significantly reduced up to 6 months after the procedure [16]. The mechanic effect of LOD on polycystic ovaries may reside in destruction of small antral and pre-antral follicles, besides stroma, resulting in a relatively long term impact on circulating levels of $\mathrm{AMH}, \mathrm{LH}$, and total and free testosterone [27]. As a result of these changes, increased ovulation and pregnancy rates have been documented after LOD in patients with PCOS. Transvaginal aspiration of small antral follicles has been proposed as a method to induce ovulation in anovulatory patients with PCO [28]: in a series of 18 patients, Mio et al. demonstrated a mean ovulation rate of $52.6 \%$ in subsequent cycles after multiple antral follicular punctures. Using a similar approach, Ferraretti et al. performed transvaginal ovarian puncture in PCOS patients to improve IVF results and named this procedure "transvaginal ovarian drilling" [29]. In a case-control study in 42 patients with PCOS who had IVM treatment before conventional IVF and 48 patients with PCOS who had not had IVM before, significantly more oocytes and embryos in subsequent IVF cycles were obtained in patients who had had
IVM treatment [6]. Moreover, Frantz et al. reported three spontaneous pregnancies in women with PCOS after transvaginal ovarian punctures for IVM. Although this is small case report series the authors concluded that multiple ovarian punctures for IVM in PCOS patients may have contributed to their pregnancy in the months following the IVM procedure [30]. However, although the above observations imply that multiple punctures for IVM may have an effect similar to that of LOD, no study has been conducted to provide a biological explanation for this effect. In the current study we examined the true impact of transvaginal puncture of minimally stimulated polycystic ovaries on the endocrine profile and conclude that the effect is only temporary: serum AMH concentrations had returned to levels before oocyte retrieval within two weeks after IVM-OR and the reduction of androgen levels had disappeared within three months after IVM-OR, at least in the subset of patients who had a follow-up at three months. This rather short-term effect compared to LOD might be explained by the relatively less invasive nature of ovarian puncture for the retrieval of immature oocytes, probably because of the absence of thermal energy. Hence, the new wave of developing small antral follicles that is observed as early as five days after IVM-OR [31] will result in restored steroidogenesis and AMH synthesis soon after the procedure.

In spite of its prospective design, the current study has several limitations. First, serial hormone analysis was restricted to the IVM cycle itself and only a small subset of patients were available for hormone profiling at three months' follow-up. After one IVM cycle, the majority of patients in this study were not pregnant and proceeded with reproductive treatment shortly after this failed treatment cycle. The observation, at least in the small subset of five patients available for follow-up, that the endocrine profile was unchanged at three months' follow-up, leads us to suggest that it is highly unlikely that the results would have been different at longer intervals. However, follow-up data were only available for five patients, which significantly limits the ability to generalize these results to a larger population.

Secondly, this study only focused on markers of the endocrine profile; the effect of ovarian puncture on ovulatory function and clinical outcomes following IVM-OR could not be investigated within the scope of this study, since the majority of patients decided to have further ART cycles shortly after the study cycle, rather than await potential effects of treatment on endocrine or ovulatory function.

Furthermore, the effect of multiple ovarian punctures for IVM on the endocrine profile described in this study has not been compared to the effect of a regular oocyte retrieval in women with PCOS undergoing a c-ART. A 
prospective study should be conducted in order to confirm the findings of the current study.

Our study cohort consisted of patients with PCOS, who were generally overweight or obese (mean BMI $30.3 \pm 9.0 \mathrm{~kg} / \mathrm{m}^{2}$ ) but who had relatively low serum levels of LH levels $(7.9 \pm 4.4 \mathrm{IU} / \mathrm{L})$ and normal markers of hyperandrogenism (TT $0.36 \pm 0.13 \mathrm{nmol} / \mathrm{L}$, FTc $5.0 \pm$ $3.6 \mathrm{pmol} / \mathrm{L}$ and SHBG $74.6 \pm 59.1 \mathrm{nmol} / \mathrm{L}$ ). In a study investigating parameters that are predictive of ovulation rates and pregnancy in 200 patients with PCOS who underwent LOD, it was shown that morbid obesity and high levels of androgens, among other parameters, are associated with low chances of response to LOD [32]. According to these findings, the patients in our study would be predicted good responders to LOD in terms of ovulatory function and clinical outcomes. Again, longterm follow-up to evaluate the effect of oocyte retrieval for IVM on ovulation rates and pregnancy could not be performed in our patients.

\section{Conclusions}

The clinical implication from the current study is that ovarian puncture for immature oocyte retrieval appears not to have a long-term effect on the endocrine profile in patients with PCOS, contrary to what is observed after LOD. The effect of ovarian puncture appears to be transient and completely recovers three months after oocyte retrieval.

The lower efficiency of IVM systems compared to gonadotropin-driven ART precludes the widespread use of current IVM protocols. Improvement of IVM culture media could substantially enhance the potential of this technology [33], especially in patients with PCOS, in whom conventional methods of ART can be cumbersome and are associated with an increased risk of OHSS. Whether ovarian puncture for IVM treatment could enhance clinical outcomes of subsequent conventional ART treatment in patients with PCOS, remains to be confirmed. The shortterm improvement of endocrine parameters related to excess follicle number and increased androgen biosynthesis in patients with PCOS might result in an increased number of oocytes and embryos in IVF cycles following IVM as observed by Agdi et al. [6]. However, the data from our study do not confirm this hypothesis.

\section{Competing interests}

The authors declare that they have no competing interests.

\section{Authors' contributions}

$\mathrm{COH}, \mathrm{NP}, \mathrm{EA}, \mathrm{LG}, \mathrm{JS}$ and MDV contributed substantially to conception and design of the study, data acquisition, analysis and interpretation. $\mathrm{COH}, \mathrm{NP}$, $E A, L G, H T$, JS and MDV contributed in drafting the article or revising it critically for important intellectual content. All authors read and approved the final manuscript.

\section{Acknowledgements}

The authors would like to thank to Johan Schiettecatte, to the clinical embryologists, laboratory technicians and nurses of the Centre for Reproductive Medicine. In vitro maturation research at the Vrije Universiteit Brussel has been granted by the Institute for the Promotion of Innovation by Science and Technology in Flanders, Project number IWT 070719 (Principle investigator: J. Smitz).

\section{Author details}

${ }^{1}$ Centre for Reproductive Medicine, UZ Brussel, Laarbeeklaan 101, 1090 Brussels, Belgium. 'Laboratory of Clinical Chemistry and Radioimmunology, UZ Brussel, Laarbeeklaan 101, 1090 Brussels, Belgium.

Received: 12 October 2013 Accepted: 1 February 2014

Published: 24 February 2014

\section{References}

1. Humaidan P, Quartarolo J, Papanikolaou EG: Preventing ovarian hyperstimulation syndrome: guidance for the clinician. Fertil Steril 2010, 94:389-400.

2. Marci R, Senn A, Dessole S, Chanson A, Loumaye E, De Grandi P, Germond M: A low-dose stimulation protocol using highly purified folliclestimulating hormone can lead to high pregnancy rates in in vitro fertilization patients with polycystic ovaries who are at risk of a high ovarian response to gonadotropins. Fertil Steril 2001, 75:1131-1135.

3. Devroey P, Polyzos NP, Blockeel C: An OHSS-free clinic by segmentation of IVF treatment. Hum Reprod 2011, 26:2596-2597.

4. Seyhan A, Ata B, Polat M, Son W-Y, Yarali H, Dahan MH: Severe early ovarian hyperstimulation syndrome following $\mathrm{GnRH}$ agonist trigger with the addition of 1500 IU hCG. Hum Reprod 2013, 28:2522-2528.

5. Huang JYJ, Chian RC, Tan SL: Ovarian hyperstimulation syndrome prevention strategies: in vitro maturation. Semin Reprod Med 2010, 28:519-531.

6. Agdi M, Zarei A, Al-Sannan B, Tulandi T, Tan SL: Effects of ovarian puncture for in vitro maturation on subsequent in vitro fertilization cycle in women with polycystic ovaries. Fertil Steril 2010, 94:381-383.

7. Farquhar C, Brown J, Marjoribanks J: Laparoscopic drilling by diathermy or laser for ovulation induction in anovulatory polycystic ovary syndrome. Cochrane Database Syst Rev 2012, 6:CD001122.

8. Rotterdam ESHRE/ASRM-Sponsored PCOS Consensus Workshop Group: Revised 2003 consensus on diagnostic criteria and long-term health risks related to polycystic ovary syndrome. Fertil Steril 2004, 18:19-25.

9. Rotterdam ESHRE/ASRM-Sponsored PCOS Consensus Workshop Group: Revised 2003 consensus on diagnostic criteria and long-term health risks related to polycystic ovary syndrome (PCOS). Hum Reprod 2004, 19:41-47.

10. De Vos M, Ortega Hrepich C, Albuz FK, Guzman L, Polyzos NP, Smitz J, Devroey P: Clinical outcome of non-hCG-primed oocyte in vitro maturation treatment in patients with polycystic ovaries and polycystic ovary syndrome. Fertil Steril 2011, 96:860-864.

11. Guzman L, Adriaenssens T, Ortega-Hrepich C, Albuz FK, Mateizel I, Devroey $P$, De Vos M, Smitz J: Human antral follicles $<6 \mathrm{~mm}$ : a comparison between in vivo maturation and in vitro maturation in non-hCG primed cycles using cumulus cell gene expression. Mol Hum Reprod 2012, 19:7-16.

12. Vermeulen A, Verdonck L, Kaufman JM: A critical evaluation of simple methods for the estimation of free testosterone in serum. $J$ Clin Endocrinol Metab 1999, 84:3666-3672.

13. Farquhar C, Lilford RJ, Marjoribanks J, Vandekerckhove P: Laparoscopic "drilling" by diathermy or laser for ovulation induction in anovulatory polycystic ovary syndrome. Cochrane Database Syst Rev 2007, Issue 3. Art. No.: CD001122. doi:10.1002/14651858.CD001122.pub3.

14. Armar NA, Lachelin GC: Laparoscopic ovarian diathermy: an effective treatment for anti-oestrogen resistant anovulatory infertility in women with the polycystic ovary syndrome. Br J Obstet Gynaecol 1993, 100:161-164.

15. Gjønnaess $\mathrm{H}$ : Late endocrine effects of ovarian electrocautery in women with polycystic ovary syndrome. Fertil Steril 1998, 69:697-701.

16. Elmashad Al: Impact of laparoscopic ovarian drilling on anti-Müllerian hormone levels and ovarian stromal blood flow using three-dimensional power Doppler in women with anovulatory polycystic ovary syndrome. Fertil Steril 2011, 95:2342-2346. 
17. Amer SAK, Gopalan V, Li TC, Ledger WL, Cooke ID: Long term follow-up of patients with polycystic ovarian syndrome after laparoscopic ovarian drilling: clinical outcome. Hum Reprod 2002, 17:2035-2042.

18. Son W-Y, Chung J-T, Herrero B, Dean N, Demirtas E, Holzer H, Elizur S, Chian RC, Tan SL: Selection of the optimal day for oocyte retrieval based on the diameter of the dominant follicle in hCG-primed in vitro maturation cycles. Hum Reprod 2008, 23:2680-2685.

19. Shalom-Paz E, Marzal A, Wiser A, Almog B, Reinblatt S, Tulandi T, Holzer H: Effects of different body mass indices on in vitro maturation in women with polycystic ovaries. Fertil Steril 2011, 96:336-339.

20. Vitek WS, Witmyer J, Carson SA, Robins JC: Estrogen-suppressed in vitro maturation: a novel approach to in vitro maturation. Fertil Steril 2013, 99:1886-1890.

21. Fanchin R, Schonäuer LM, Righini C, Frydman N, Frydman R, Taieb J: Serum anti-Müllerian hormone dynamics during controlled ovarian hyperstimulation. Hum Reprod 2003, 18:328-332.

22. La Marca A, Malmusi S, Giulini S, Tamaro LF, Orvieto R, Levratti P, Volpe A: Anti-Müllerian hormone plasma levels in spontaneous menstrual cycle and during treatment with FSH to induce ovulation. Hum Reprod 2004, 19:2738-2741.

23. Baarends WM, Hoogerbrugge JW, Post M, Visser JA, De Rooij DG, Parvinen M, De Rooij DG, Parvinen M, Themmen AP, Grootegoed JA: Anti-müllerian hormone and anti-müllerian hormone type II receptor messenger ribonucleic acid expression during postnatal testis development and in the adult testis of the rat. Endocrinology 1995, 136:5614-5622.

24. Pellatt L, Hanna L, Brincat M, Galea R, Brain H, Whitehead S, Mason H: Granulosa cell production of anti-Müllerian hormone is increased in polycystic ovaries. J Clin Endocrinol Metab 2007, 92:240-245.

25. Smitz J, Andersen AN, Devroey P, Arce JC, MERIT Group: Endocrine profile in serum and follicular fluid differs after ovarian stimulation with HP-hMG or recombinant FSH in IVF patients. Hum Reprod 2007, 22:676-687.

26. Amer SA, Li TC, Ledger WL: The value of measuring anti-Mullerian hormone in women with anovulatory polycystic ovary syndrome undergoing laparoscopic ovarian diathermy. Hum Reprod 2009, 24:2760-2766.

27. Amer SAKS, Banu Z, Li TC, Cooke ID: Long-term follow-up of patients with polycystic ovary syndrome after laparoscopic ovarian drilling: endocrine and ultrasonographic outcomes. Hum Reprod 2002, 17:2851-2857.

28. Mio Y, Toda T, Tanikawa M, Terado H, Harada T, Terakawa N: Transvaginal ultrasound-guided follicular aspiration in the management of anovulatory infertility associated with polycystic ovaries. Fertil Steril 1991, 56:1060-1065.

29. Ferraretti AP, Gianaroli L, Magli MC, lammarrone E, Feliciani E, Fortini D: Transvaginal ovarian drilling: a new surgical treatment for improving the clinical outcome of assisted reproductive technologies in patients with polycystic ovary syndrome. Fertil Steril 2001, 76:812-816.

30. Frantz A, Ferreira M, Hoher M, Bos-Mikich A: Spontaneous pregnancies after ovarian punctures for in vitro maturation in women with polycystic ovary syndrome. Rev Bras Ginecol Obstet 2009, 31:138-141.

31. Elizur SE, Son W-Y, Clarke H, Morris D, Gidoni Y, Demirtas E, Tan SL: A unique biological in-vivo model to evaluate follicular development during in-vitro maturation treatment. Reprod BioMed Online 2011, 22:257-262

32. Amer S: Ovulation induction using laparoscopic ovarian drilling in women with polycystic ovarian syndrome: predictors of success. Hum Reprod 2004, 19:1719-1724.

33. Albuz FK, Sasseville M, Lane M, Armstrong DT, Thompson JG, Gilchrist RB: Simulated physiological oocyte maturation (SPOM): a novel in vitro maturation system that substantially improves embryo yield and pregnancy outcomes. Hum Reprod 2010, 25:2999-3011.

\section{doi:10.1186/1477-7827-12-18}

Cite this article as: Ortega-Hrepich et al:: The effect of ovarian puncture on the endocrine profile of PCOS patients who undergo IVM.

Reproductive Biology and Endocrinology 2014 12:18. 